\title{
Sound Field Diffusivity Assessment in Non-Stationary State by Measuring Nonuniformity of Sound Decay Curve
}

\author{
K. LEO*
}

\author{
Faculty of Physics and Applied Mathematics, Technical University of Gdańsk \\ G. Narutowicza 11/12, 80-233 Gdańsk, Poland
}

\begin{abstract}
Methods of sound diffusion assessment presented in literature require information on directional properties of the sound field in stationary state. These methods are complicated and not often applicable in measurement practice. Measurement of the sound field diffusivity during its formation can be assessed from sound level decay curve deviation from a linear shape. With this method, the degree of diffusivity has been determined in three rooms. Measurements were performed in rooms differing in volumes and sound absorption. Changes in sound decay uniformity were noted, which can be translated into diffusion coefficient changes. Results indicate that diffusion increases as room absorption decreases. Diffusion does not depend on room volume. Changes of sound decay uniformity follow trends that are well described by power function.
\end{abstract}

PACS: 43.55.Br, 43.55.Cs

\section{Introduction}

Sound diffusion measurements have been performed since the early fifties of the 20th century, when Schroeder presented equations describing diffusion [1]. Bodlund's research resulted in cross-correlation coefficient for the sound pressure [2]. Other authors describe sound diffusion at a given observation point as the degree of sound energy uniformity coming from various directions $[3,4]$. The observation point can be regarded as a sphere divided into planes facing various directions, with sound energy in acoustic stationary field measured when passing through each of them. Spatial nonuniformity of sound energy is calculated by dividing the total of the sound energy which came through individual planes by the energy of isotropic sound field. Detailed description of diffusion in a room in its modal state was given by Nelisse et al. [3]. Modal density is investigated in order to achieve adequate diffusion. This work describes a method based on geometric approach to sound propagation in a room. Loutridris [5] introduced new diffusion measure based on the assumption that impulse response can be regarded as multifractal signal. Impulse response is regarded as multiplicative, multifractal signal due to the observation that cascades of randomly diffused reflections generate multifractal structured reflections. Thus singularity spectrum width is a stable measure of diffusion in small rooms. Singularity spectrum width of ideally diffused field equals zero.

2. Two methods of sound diffusion measurement 2.1. Spatial method in stationary field

Shroeder and others investigated 3D stationary sound field $[6,7]$ exploring directional properties of diffused

* e-mail: krzysztof.leo@gmail.com sound field. One-dimensional sound diffusion index is calculated for all measured directions. Furduyev and Tun described the method of measuring diffusion index in $2 \mathrm{D}$ field [8]. In this method, a directional microphone rotating in a horizontal plane is applied. In a perfectly diffused field, energy value is constant for all the measured directions. Sound diffusion index in stationary state measures energy response of the room for all horizontal directions, then the response is averaged for one-dimensional diffusion index. For this purpose, polar diagram of the microphone output is recorded in the room and in an anechoic chamber, with stationary noise emitted by a loudspeaker. Sound diffusion index $d_{\mathrm{s}}$ in the stationary state is defined as:

$$
d_{\mathrm{s}}=1-\frac{A_{\text {measur }}}{A_{\text {ref }}} .
$$

$A_{\text {ref }}, A_{\text {measur }}$ - area of polar diagram bounding sound energy measured in anechoic chamber and in the room, respectively.

If stationary noise is considered as a series of individual impulses, sound decay phenomena excited by them overlap one another. Sound response of the room is then considered as time superposition of individual sound decays (Fig. 1).

Spatial methods require advanced measurement techniques e.g. rotational microphone or spherical microphone. The need for a simple method of comparable accuracy arises. The method should facilitate the room impulse response, because of the ease of measurement and comparison of the already measured rooms.

\subsection{Time method in non-stationary field}

Room impulse response is considered as a non-stationary state of acoustic field. An analysis of the subtle structure of impulse response reveals that the state of sound dispersion changes during sound field formation. As impulse decay begins, early energy consisting of direct sound and strong, discrete reflections induces 


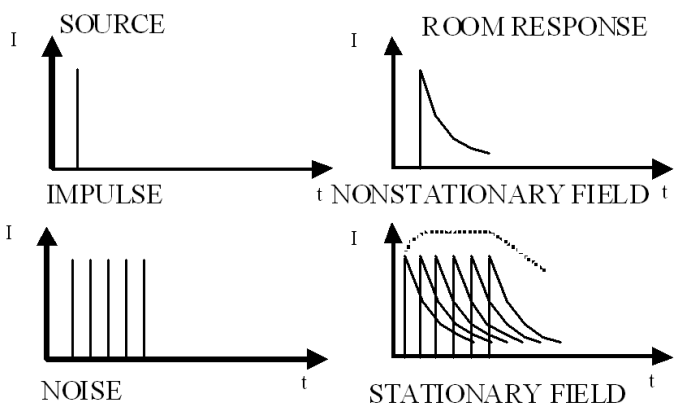

Fig. 1. Stationary and non-stationary field — signal from the source and comparison of the room's responses. In stationary field, room's response is time superposition of individual impulse responses. Build-up process, stationary state and decay process shown with dotted line. Diffused field in both fields is assumed. $I$ - sound intensity, $\mathrm{W} / \mathrm{m}^{2}, t$ - time, $\mathrm{s}$.

high nonuniformity of sound level decay. Later on, late energy consisting of many diffused reflections leads to uniformity of decay. Measurement of nonuniformity of sound level decay enables us to determine sound diffusion in the room [9]. Figure 2 shows how different areas of sound level decay are built in this work. The degree of nonuniformity can be calculated from Eq. (2).

Nonuniformity index $S_{\mathrm{a}}$ of sound level decay equals [9]:

$$
S_{\mathrm{a}}=\frac{A_{\text {real }}}{A_{\text {ideal }}} .
$$

By analogy to Eq. (1), sound diffusion index $d_{\mathrm{t}}$ in non-stationary state equals

$$
d_{\mathrm{t}}=\frac{A_{\text {ideal }}-A_{\text {real }}}{A_{\text {ideal }}}=1-\frac{A_{\text {real }}}{A_{\text {ideal }}}=1-S_{\mathrm{a}},
$$

where $A_{\text {ideal }}$ and $A_{\text {real }}$ is energy of sound level decay of perfectly diffused field and the measured field, respectively.

\section{Estimation of sound diffusion}

\subsection{The method}

Acoustic measurements in three rooms before and after acoustic adaptation have been performed. Rooms varying in volume, shape and sound absorption were chosen. Typical acoustic treatment was performed in each room. Details of acoustic adaptation are described in Tables I and II.

TABLE I

\begin{tabular}{|c|c|c|c|}
\hline & Classroom 1 & Classroom 2 & Church \\
\hline abbreviation & $\mathrm{S} 14$ & S72 & JEZ \\
\hline volume $\left[\mathrm{m}^{3}\right]$ & 195 & 264 & 19341 \\
\hline dimensions $[\mathrm{m}]$ & $7.62 \times 7.75 \times 3.3$ & $6.5 \times 12.5 \times 3.3$ & $35 \times 32 \times 28$ \\
\hline $\begin{array}{l}\text { reverberation time } T_{30}[\mathrm{~s}] \text { (before } \\
\text { acoustic adaptation, } f=1 \mathrm{kHz} \text { ) }\end{array}$ & 0.8 & 1.7 & 6.78 \\
\hline indoor materials & $\begin{array}{l}\text { lime plaster, } \\
\text { windows, parquet }\end{array}$ & $\begin{array}{l}\text { lime plaster, } \\
\text { windows, linoleum }\end{array}$ & $\begin{array}{l}\text { lime plaster, windows, } \\
\text { stone floor, wooden pews }\end{array}$ \\
\hline $\begin{array}{c}\text { place and type } \\
\text { of additional } \\
\text { acoustic adaptation: }\end{array}$ & $\begin{array}{c}\text { ceiling: } \\
\text { Ecophon Gedina }\end{array}$ & $\begin{array}{c}\text { ceiling: } \\
\text { Ecophon Master Alpa }+ \\
\text { Master Gamma } \\
\text { rear wall: } \\
\text { Ecophon Panel } \\
\text { (3 setups of adaptation) }\end{array}$ & $\begin{array}{c}\text { walls: } \\
\text { perforated } \\
\text { plasterboard }\end{array}$ \\
\hline
\end{tabular}

Investigated rooms, each room was measured without and with acoustic adaptation.

In each room impulse responses were registered by multilength sequence (MLS) technique with a pair of directional GENELEC 8020A speakers and an omnidirectional microphone. One measurement point in reverberant field was chosen in each room.

Nonuniformity of sound level decay was estimated according to the method described in (1.2) above. Nonuniformity of impulse responses was determined by taking into account the increasing dynamic step $\Delta L$. Dynamic step is increased by lowering the value of $L_{2}$ while $L_{1}$ remains constant — see Fig. 2 .

\subsection{The results}

Nonuniformity of sound level decay vs. dynamic step is well described with power function (see Fig. 3). Dynamic step as a measure of sound energy decay has been chosen, because it enables a comparison of nonuniformity of decay in rooms of different reverberation times. Dynamic step could be easily transformed into time value, but the comparison of decay nonuniformity in different rooms would not be so explicit. Power trend line coefficients are shown in Table II. 
TABLE II

Power trend line coefficients $(a, b)$ describing the emergence of nonuniformity of room impulse response $\left(y=a x^{b}\right)$. Coefficients are shown for broadband. Predictions of $S_{\mathrm{a}}$ value for $(-5 \mathrm{~dB} ;-65 \mathrm{~dB})$ and $(-5 \mathrm{~dB} ;-95 \mathrm{~dB})$ dynamic step of echogram are calculated according to trend line coefficients. Surfaces in

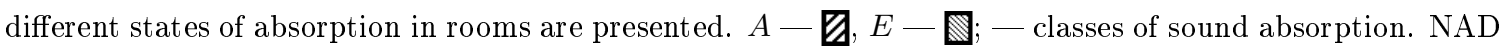
— not adapted, AD - adapted, S14 - classroom 1, S72 - classroom 2, JEZ - church.

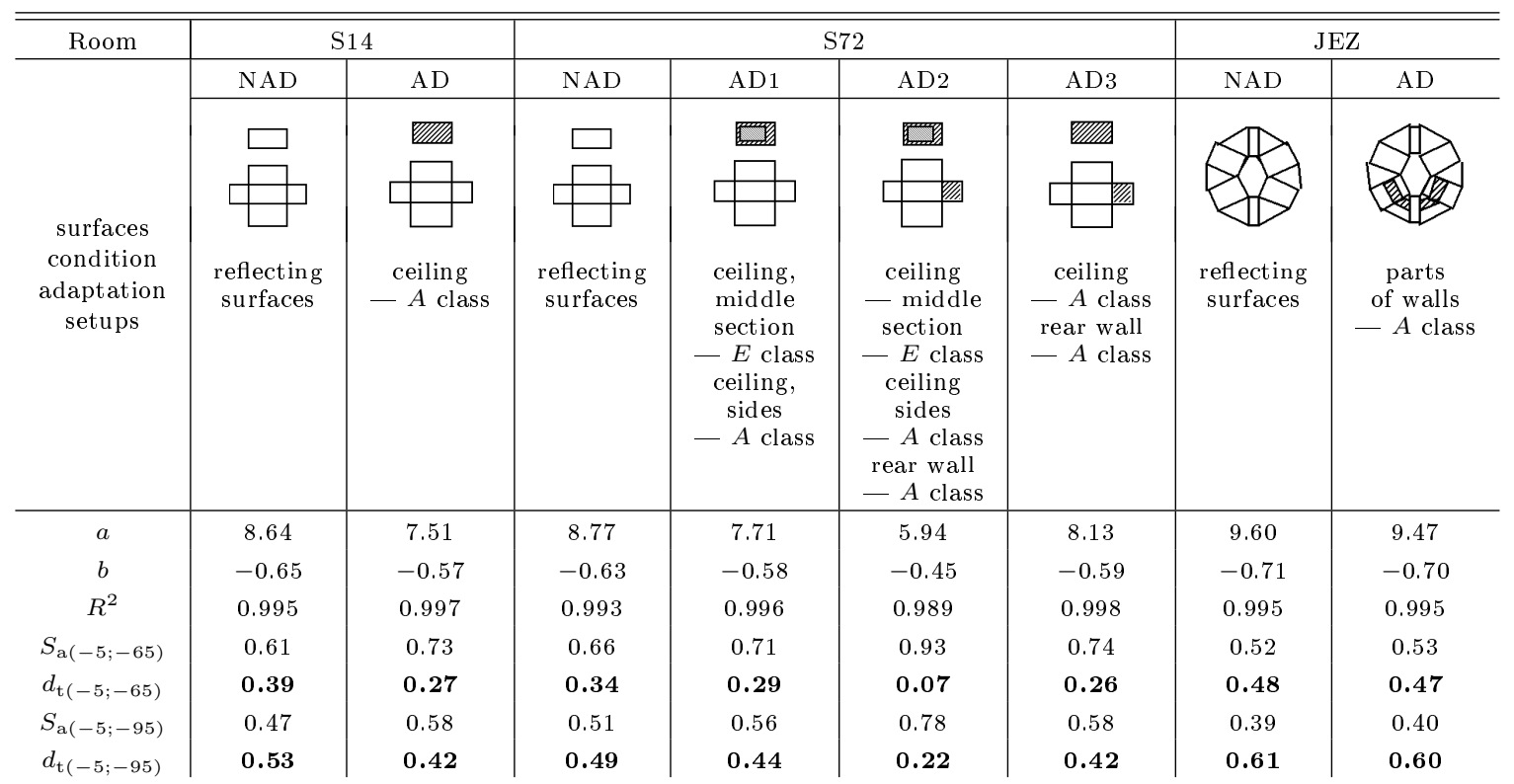

\subsection{Discussion}

- As shown in Fig. 3, the degree of nonuniformity is a descending function of the dynamic step. Increasing value of diffusion in non-stationary state can be calculated with Eq. (3). The increase in the diffusion in these rooms is proportional to the nonuniformity of sound level decay. In the early stages of impulse response diffusion process, nonuniformity can reach values larger than 1 . A calculation of diffusion according to Eq. (3) can give misleading results of negative diffusion index values. Thus it is supposed that the value of diffusion index in non-stationary field approaches the value of diffusion index in stationary field as dynamic step is increased. The duration of the diffusing process in non-stationary state is infinite. In measurement practice, the largest possible time window or the dynamic step of impulse response should be considered.

- Power trend line ( $a$ and $b$ coefficients) well describes changes of sound nonuniformity in the room. The coefficient $a$ is inversely proportional to the speed of diffusion changes. Both $a$ and $b$ describe degree of diffusion in stable state according to $d_{\mathrm{t}}$ in Eq. (1), when the value of dynamic step is very large. Approximation with trend line enables the calculation
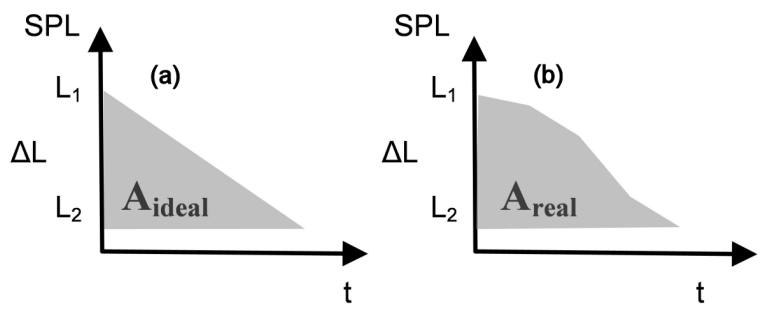

Fig. 2. Sound decay curve in diffuse (a) and non diffuse (b) sound field (sound level vs. time), dynamic step $\Delta L$ indicated as difference between values $L_{1}$ and $L_{2}$. Nonuniformity of sound decay can be calculated at a given dynamic step. Different values of dynamic step obtained by lowering $L_{2}$ value, while $L_{1}$ remains constant can give different values of nonuniformity. $A_{\text {ideal }}$ and $A_{\text {real }}$ is bounded by level $L_{1}$ and $L_{2}$ sound level decay energy. $A_{\text {ideal }}$ and $A_{\text {real }}$ is energy of sound level decay of perfectly diffused field and the measured field, respectively.

of diffusion index in larger dynamic step — value not available in practical measurements. While the dynamic step reaches infinity, index $d_{\mathrm{t}}$ can be regarded as equal to $d_{\mathrm{s}}$.

- The calculated values of diffusion index $d_{\mathrm{t}}$ in Table II are rising when dynamic step is increased. This is consistent with the expectations. 


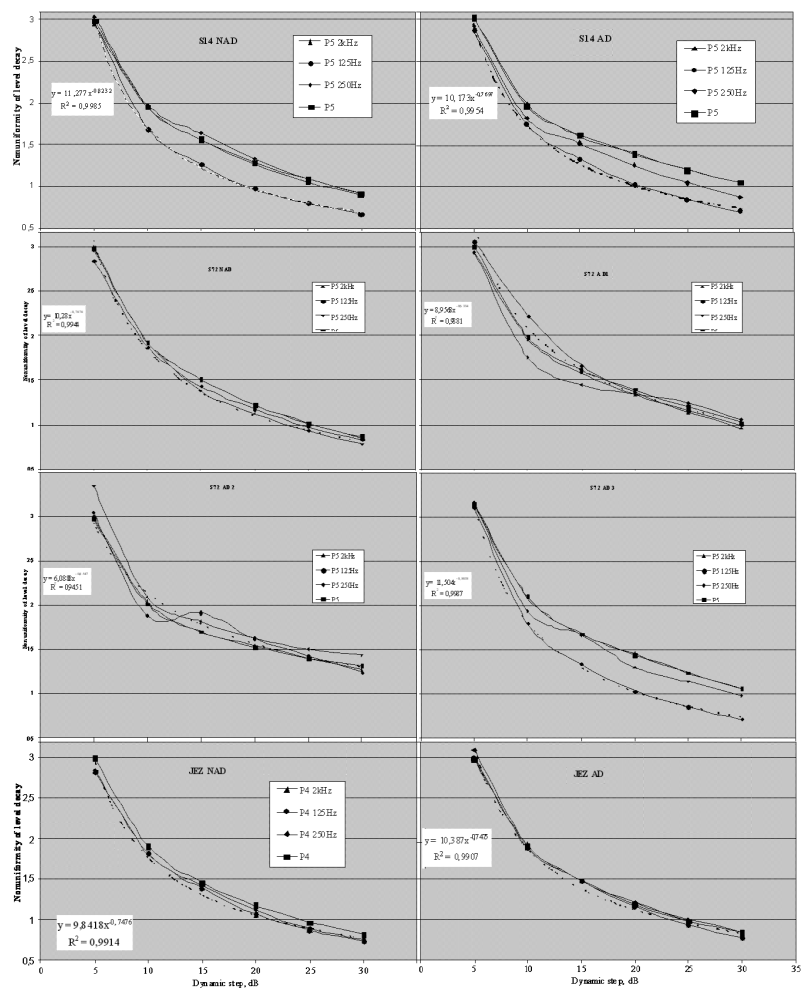

Fig. 3. Nonuniformity of sound level decay (according to Eq. (2)) vs. increasing dynamic step, $\Delta L$ for different rooms, with and without acoustic adaptation. NAD not adapted room, AD - adapted room. Fitting trend lines $(---, 125 \mathrm{~Hz})$ as well as their equations and $R^{2}$ coefficient values are shown.

- Different shape of nonuniformity curves (Fig. 3) reveals different speed of diffusion formation in the rooms. Where only one surface has been adapted, diffusion forming rate is smaller than in a nonadapted room.
- Greater dispersion in the frequency of nonuniformity and its forming rate is noted in small rooms (Fig. 3), especially in adapted rooms.

\section{Summary}

Measuring diffusion in non-stationary state is equivalent to stationary state measurements. This has practical consequences, i.e. it simplifies measurement method. Diffusion, expressed either with $d_{\mathrm{s}}$ or $d_{\mathrm{t}}$ coefficients, can be calculated from the linearity of sound level decay. Measurements performed in rooms varying in sound absorption and material placement show significant differences in sound diffusion index $d_{\mathrm{t}}$. More measurements are needed to clarify the correlation between values of absorption coefficient, placement of sound absorption materials, room shape and sound diffusion indices. The correlation between various diffusion measures should be established.

\section{References}

[1] M. Schroeder, J. Acoust. Soc. Am. 31, 1407 (1959).

[2] K. Bodlund, J. Sound Vibrat. 44, 191 (1976).

[3] H. Nelisse, J. Nicolas, J. Acoust. Soc. Am. 101, 3517 (1997).

[4] F. Jacobsen, T. Roisin, J. Acoust. Soc. Am. 108, 204 (2000).

[5] S.J. Loutridris, J. Acoust. Soc. Am. 125, 1498 (2009).

[6] B.N. Gover, J.G. Ryan, M.R. Stinson, J. Acoust. Soc. Am. 116, 2138 (2004).

[7] A. Kulowski, Appl. Acoust. 32, 93 (1991).

[8] W. Furduyev, C. Tun, Sov. Phys. - Acoustics 6, 103 (1960).

[9] N.F. Spring, K.E. Randall, $B B C R \& D$ Report, BBC, London 1969. 